\title{
Spectral Functions for Gauge Fields in Rindler-Like Spaces
}

\section{Andrey A. Bytsenko}

Depto. de Física, Univ. Estadual de Londrina, Paraná, Brazil E-mail: abyts@uel.br

\section{Antonio Edson Gonçalves}

Depto. de Física, Univ. Estadual de Londrina, Paraná, Brazil E-mail: goncalve@uel.br

\section{Vanderlei dos Santos Mendes*}

Depto. de Física, Univ. Estadual de Londrina, Paraná, Brazil E-mail: vsmendes@uel.br

A class of conformal deformations of Rindler-like spaces is analyzed. We study the spectral properties of the Laplace operators associated with $p$-forms and acting in these spaces and in their spatial sections. The spectral density of continuum spectrum and the spectral zeta functions related to the abelian $p$-forms in real compact hyperbolic manifolds are obtained.

Fourth International Winter Conference on Mathematical Methods in Physics 09 - 13 August 2004

Centro Brasileiro de Pesquisas Fisicas (CBPF/MCT), Rio de Janeiro, Brazil

\footnotetext{
* Speaker.
} 


\section{Introduction}

In this paper we consider a class of conformal deformations of Rindler-like spaces, whose spatial sections have metric conformally related to the metric of hyperbolic spaces, and the spectral properties of the Laplace operators acting on these spaces. The real hyperbolic spaces play important role in supergravity [1], superstring theory [2, 3] and cosmology [4, 5]. The finite temperature effects for massive scalar fields in Rindler spaces, in its conformal connection to hyperbolic spaces, have been considered in [6]. Here we analize gauge theories based on abelian $p$-forms and calculate in the Rindler case (non-compact manifold) the spectral datas of Laplacians and the measure density useful for the trace of tensor kernels. The spectral zeta functions of real compact hyperbolic spaces are calculated explicitly using the Fried trace formula.

\section{Spectral functions of space forms $M=\mathbb{R}^{+} \times X^{N}$}

Let $M$ be a $D=(N+2)$-dimensional space and let $d s^{2}=g_{00}(\mathbf{x})\left(d x^{0}\right)^{2}+g_{i j}(\mathbf{x}) d x^{i} d x^{j}, \mathbf{x}=$ $\left\{x^{j}\right\} i, j=1, \ldots, N+1$. For the class of conformal deformations of the Riemannian metric $g_{\mu v}$ the following relation holds:

$$
\widetilde{g}_{\mu v}(\mathbf{x})=e^{2 \sigma(\mathbf{x})} g_{\mu v}(\mathbf{x}), \quad \sigma(\mathbf{x}) \in C^{\infty}(M) .
$$

Let us consider static spaces admiting canonical horizons and having the topology of the form $\mathbb{R} \times \mathbb{R}^{+} \times X^{N}$. The metric reads

$$
d s^{2}=-b^{2} x^{2} d x_{0}^{2}+d x^{2}+d \Omega_{N}^{2}
$$

where $b$ is a constant factor, and $d \Omega_{N}^{2}$ is the spatial metric related to the $N$-dimensional manifold $X^{N}$. If $X^{N} \equiv \mathbb{R}^{N}$, one has to deal with the Rindler space. If $X^{N} \equiv S^{N}, b=(D-3) /\left(2 R_{H}\right)$ and $R_{H}$ is the Schwarzschild radius of a black hole, then one is dealing with a space which approximates, near the horizon and in the large mass limit, a $D$-dimensional black hole [6]. In the Euclidean sector the metric (2.2) may be written in the conformally related form:

$$
d \widetilde{s}^{2}=d \tau^{2}+x^{-2}\left(d x^{2}+d \Omega_{N-1}^{2}\right),
$$

where $\sigma(\mathbf{x})=-(1 / 2) \log g_{00}, d \tau=i b d x^{0}, x^{-2}$ is the conformal factor. Associated to the conformally deformed metric (2.3) we get the quantities $d V=x^{-N-1} d x d V_{N}$, and

$$
\begin{aligned}
& L^{(N+1, \widetilde{g})}=-\mathfrak{L}^{(N+1, \widetilde{g})}-\rho_{0}^{2}+C x^{2}, \\
& \mathfrak{L}^{(N+1, \widetilde{g})}=-x^{2} \partial_{x}^{2}+(N-2) x \partial_{x}+x^{2} \mathfrak{L}^{(N, g)},
\end{aligned}
$$

where $\mathfrak{L}^{(N, g)}$ is the Laplace operator on the manifold $X^{N}, d V_{N}$ its invariant measure, $\rho_{0}=(N-1) / 2$, and the real constant $C$ depends on the scalar curvature.

Let us search for the spectral properties of the elliptic operator $L^{(N+1, \widetilde{g})}$.

Theorem 1. (A. A. Bytsenko, G. Cognola and S. Zerbini [6]]) For any suitable function $F\left(L^{(N+1, \widetilde{g})}\right)$ the following formulas hold:

$$
\begin{aligned}
& \operatorname{Tr} F\left(L^{(N+1, \widetilde{g})}\right)=\int_{0}^{\infty} F\left(s^{2}\right) \mu_{I}(s) d s \\
& \mu_{I}(s)=\mu(s) \int_{0}^{\infty} \sum_{\alpha}\left|K_{i s}\left(x \lambda_{\alpha}\right)\right|^{2} x^{-1} d x
\end{aligned}
$$


where $K_{i s}(y)$ are the Bessel functions of imaginary argument and $\left\{\lambda_{\alpha}\right\}$ is the set of eigenvalues of the operator $L^{(N+1, \widetilde{g})}$.

Here we derive a general expression for $\mu_{I}(r)$ by making use of Eq. (2.6).

Proposition 1. Let $\mathcal{K}_{t}=\exp \left(-t L^{(N+1, \widetilde{g})}\right)$ be the heat kernel of the operator $L^{(N+1, \widetilde{g})}, \mathcal{K}_{\ell}\left(L^{(N+1, \widetilde{g})}\right)$ are the Seeley-De Witt coefficients in the kernel expansion, and $\varepsilon$ is a horizon cutoff parameter in integrating over the space coordinates in Eq. (2.6). Then we have:

$$
\begin{aligned}
& \mu_{I}(r)=\sum_{\ell=0}^{\left[\frac{N-1}{2}\right]} \frac{\mathcal{K}_{2 \ell}\left(\mathfrak{L}_{p}^{(N, \widetilde{g})}\right) \Phi(r, N+1-2 \ell)}{N-2 \ell}\left(4 \pi \varepsilon^{-2}\right)^{(N-2 \ell) / 2}+\frac{1}{2 \pi} \zeta\left(0 \mid \mathfrak{L}^{(N, g)}\right) \\
& \times\left[\left.\frac{d}{d s} \log \zeta\left(s \mid \mathfrak{L}^{(N, g)}\right)\right|_{s=0}+\psi(i r)+\psi(-i r)-2 \log \left(\frac{\varepsilon}{2}\right)-\pi \delta(r)\right]
\end{aligned}
$$

where

$$
\Phi(r, N+1)=\mu(r) \operatorname{Vol}\left(S^{N-1}\right)\left(\frac{x}{2 \pi}\right)^{N} \int_{0}^{\infty}\left|K_{i r}(x s)\right|^{2} s^{N-1} d s,
$$

$\operatorname{Vol}\left(S^{N-1}\right)$ is the volume of the $(N-1)$-dimensional sphere, $\left[\frac{N-1}{2}\right]$ is the integer part of the number $(N-1) / 2, \psi(z)$ is the logarithmic derivative of $\Gamma-$ function and $\delta(r)$ is the Dirac delta function. For odd $N, \zeta\left(0 \mid \mathfrak{L}^{(N, g)}\right)$ is vanishing and so the last term in Eq. (2.7) disappears.

Proof. We can use the Mellin-Barnes representation for the Bessel function of imaginary argument [7] $K_{i r}^{2}\left(x \lambda_{\alpha}\right)=(-16 \pi)^{-1 / 2} \int_{\Re_{s>1}} \Gamma(s+i r) \Gamma(s-i r) \Gamma(s)[\Gamma(s+1 / 2)]^{-1}\left(x \lambda_{\alpha}\right)^{-2 s} d s$, and observe that, for $\Re s>N / 2$, the sum over $\alpha$ gives $\sum_{\alpha} \lambda_{\alpha}^{-2 s}=\zeta\left(s \mid \mathfrak{L}^{(N, g)}\right)$. Thus,

$$
\mu_{I}(r)=\frac{\mu(r)}{8 \sqrt{-\pi}} \int_{\Re_{s=c>N / 2}} \frac{\Gamma(s+i r) \Gamma(s-i r) \Gamma(s) \zeta\left(s \mid \mathfrak{L}^{(N-1, g)}\right)}{s \Gamma(s+1 / 2) \varepsilon^{2 s}} d s .
$$

To make the integral we consider the rectangular contour $\mathcal{C}:=\{\Re s=c, \mathfrak{I} s=a, \Re s=-c, \mathfrak{I} s=-a\}$ and observe that the two horizontal paths $\mathfrak{I} s= \pm a$ give a vanishing contribution in the limit $a \rightarrow \infty$, as well as the path $\Re s=-c$ in the limit $\varepsilon \rightarrow 0$. Also the poles in the strip $-c<\Re s<0$ give a vanishing contribution as soon as $\varepsilon \rightarrow 0$. Then we have to take into consideration only the poles of the integrand in Eq. (2.9) in the half-plane $\Re s \geq 0$. Such a function has simple poles at the points $s=0, s=-n \pm i r$ and $s=(N-n) / 2(n \geq 0)$. If $D$ is even, that is $N$ is odd, $s=0$ is a double pole. It is clear that all poles with $\Re s>0$ give rise to divergences, the number of them depending on $N$, while the poles at $s=0$ and $s= \pm i r$ give rise to finite contributions. Thus the result (2.7) follows from Eq. (2.9).

\section{The trace formula}

Let $\omega_{p}, \varphi_{p}$ be exterior differential $p$-forms; then, the invariant inner product is defined by $\left(\omega_{p}, \varphi_{p}\right) \stackrel{\text { def }}{=} \int_{X^{N}} \omega_{p} \wedge * \varphi_{p}$. The following properties for operators and forms hold: $d d=\delta \delta=0$, $\delta=(-1)^{N p+N+1} * d *, * * \omega_{p}=(-1)^{p(N-p)} \omega_{p}$. The operators $d$ and $\delta$ are adjoint to each other with respect to this inner product for $p$-forms: $\left(\delta \omega_{p}, \varphi_{p}\right)=\left(\omega_{p}, d \varphi_{p}\right)$. In quantum field theory 
the Lagrangian associated with $\omega_{p}$ takes the form: $d \omega_{p} \wedge * d \omega_{p}$ (gauge field), and $\delta \omega_{p} \wedge * \delta \omega_{p}$ (cogauge field). These Lagrangians provide a possible representation of tensor fields or generalized abelian gauge fields. The two representations of tensor fields are not completely independent, because of the well-known duality property of exterior calculus which gives a connection between star-conjugated gauge and co-gauge tensor fields [8]. The gauge $p$-forms are mapped into the co-gauge $(N-p)$-forms under the action of the Hodge $*$ operator.

- The results (2.4), (2.7) can easy be generalized for the case of the Laplacians $\mathfrak{L}_{p}^{(N, g)}$ acting on $p$-forms.

Let $X_{\Gamma}=X^{N}$ be a $N$-dimensional real compact hyperbolic space with universal covering $Y$ and fundamental group $\Gamma$. Then we can represent $Y$ as the symmetric space $G / K$, where $G=S O_{1}(N, 1)$ and $K=S O(N)$ is a maximal compact subgroup of $G$. We regard $\Gamma$ as a discrete subgroup of $G$ acting isometrically on $Y$, and we take $X_{\Gamma}$ to be the quotient space by that action: $X_{\Gamma}=\Gamma \backslash Y=$ $\Gamma \backslash G / K$. Let $\tau$ be an irreducible representation of $K$ on a complex vector space $V_{\tau}$, and form the induced homogeneous vector bundle $G \times{ }_{K} V_{\tau}$ (the fiber product of $G$ with $V_{\tau}$ over $K$ ) over $Y$. Restricting the $G$ action to $\Gamma$ we obtain the quotient bundle $E_{\tau}=\Gamma \backslash\left(G \times_{K} V_{\tau}\right) \rightarrow X_{\Gamma}$. The natural Riemannian structure on $Y$ (therefore on $X_{\Gamma}$ ) induced by the Killing form $($,$) of G$ gives rise to a connection Laplacian $\mathfrak{L}_{p}^{(N, g)}$ on $E_{\tau}$. Let $\sigma_{p}$ be the natural representation of $S O(2 k-1)$ on $\Lambda^{p} \mathbb{C}^{2 k-1}$, and $\mu_{\sigma_{p}(r)}$ be the corresponding Harish-Chandra-Plancherel density (given for a suitable normalization of the Haar measure $d x$ on $G)$. Let $\operatorname{Vol}(\Gamma \backslash G)$ will denote the integral of the constant function 1 on $\Gamma \backslash G$ with respect to the $G$-invariant measure on $\Gamma \backslash G$ induced by $d x$. We can apply the version of the trace formula:

Theorem 2. (D. Fried [9]) For $0 \leq p \leq N-1$ the trace formula applied to kernel $\mathcal{K}_{t}=e^{-t \mathfrak{L}_{p}^{(N, g)}}$ holds:

$$
\operatorname{Tr}\left(e^{-t \mathfrak{L}_{p}^{(N, g)}}\right)=I_{\Gamma}^{(p)}\left(\mathcal{K}_{t}\right)+I_{\Gamma}^{(p-1)}\left(\mathcal{K}_{t}\right)+H_{\Gamma}^{(p)}\left(\mathcal{K}_{t}\right)+H_{\Gamma}^{(p-1)}\left(\mathcal{K}_{t}\right),
$$

where $I_{\Gamma}^{(p)}\left(\mathcal{K}_{t}\right), H_{\Gamma}^{(p)}\left(\mathcal{L}_{t}\right)$ are the identity and hyperbolic orbital integrals respectively. In the above formula

$$
\begin{aligned}
& I_{\Gamma}^{(p)}\left(\mathcal{K}_{t}\right) \stackrel{\text { def }}{=} \frac{\chi(1) \operatorname{Vol}(\Gamma \backslash G)}{4 \pi} \int_{\mathbb{R}} \mu_{\sigma_{p}}(r) e^{-t\left(r^{2}+b^{(p)}+\left(\rho_{0}-p\right)^{2}\right)} d r \\
& H_{\Gamma}^{(p)}\left(\mathcal{K}_{t}\right) \stackrel{\text { def }}{=} \frac{1}{\sqrt{4 \pi t}} \sum_{\gamma \in C_{\Gamma}-\{1\}} \frac{\chi(\gamma)}{j(\gamma)} t_{\gamma} C(\gamma) \chi_{\sigma_{p}}\left(m_{\gamma}\right) e^{-t\left(b^{(p)}+\left(\rho_{0}-p\right)^{2}\right)-t_{\gamma}^{2} / 4 t}
\end{aligned}
$$

In Eq. (3.3) $C_{\Gamma} \subset \Gamma$ is a complete set of representations in $\Gamma$ of its conjugacy classes, $C(\gamma)$ is a well defined function on $\Gamma-\{1\}$ (for more details see [10, 6, 11, 12]), $b^{(p)}$ are real constants, and $\chi_{\sigma}(m)=\operatorname{trace}(\sigma(m))$ is the character $\sigma$ for $m \in S O(2 k-1)$.

The spectral zeta function related to the Laplace operator $\mathfrak{L}_{p}^{(N, g)}$ can be represented by the inverse Mellin transform of the heat kernel $\mathcal{K}_{t}$. Using the Fried formula, we can write the zeta function as a sum of contributions:

$$
\begin{aligned}
\zeta\left(s \mid \mathfrak{L}_{p}^{(N, g)}\right) & =\frac{1}{\Gamma(s)} \int_{0}^{\infty}\left(I_{\Gamma}^{(p)}\left(\mathcal{K}_{t}\right)+I_{\Gamma}^{(p-1)}\left(\mathcal{K}_{t}\right)+H_{\Gamma}^{(p)}\left(\mathcal{K}_{t}\right)+H_{\Gamma}^{(p-1)}\left(\mathcal{K}_{t}\right)\right) t^{s-1} d t \\
& \equiv \zeta_{I}^{(N)}(s, p)+\zeta_{I}^{(N)}(s, p-1)+\zeta_{H}^{(N)}(s, p)+\zeta_{H}^{(N)}(s, p-1) .
\end{aligned}
$$


Proposition 2. The identity and hyperbolic components of the spectral zeta function can be presented in the form:

$$
\begin{aligned}
\zeta_{I}^{(2 k)}(s, p) & =\frac{V_{\Gamma} C_{2 k}^{(p)}}{\Gamma(s)} \sum_{\ell=0}^{k-1} a_{2 \ell, 2 k}^{(p)}\left[\frac{\Gamma(\ell+1) \Gamma(s-\ell-1)}{\alpha_{p}^{2 s-2 \ell-2}}+\sum_{n=0}^{\infty} \frac{\xi_{n \ell} \Gamma(s+n)}{\alpha_{p}^{2 s+2 n}}\right] \\
\zeta_{I}^{(2 k+1)}(s, p) & =\frac{V_{\Gamma} C_{2 k+1}^{(p)}}{\Gamma(s)} \sum_{\ell=0}^{k} a_{2 \ell, 2 k+1}^{(p)} \int_{0}^{\infty} t^{s-1} d t \int_{\mathbb{R}} e^{-t\left(r^{2}+\alpha_{p}^{2}\right)} r^{2 \ell} d r \\
& =\frac{V_{\Gamma} C_{2 k+1}^{(p)}}{\Gamma(s)} \sum_{\ell=0}^{k} a_{2 \ell, 2 k+1}^{(p)} \Gamma(\ell+1 / 2) \Gamma(s-\ell-1 / 2) \alpha_{p}^{-2 s+2 \ell+1}, \\
\zeta_{H}^{(N)}(s, p) & =\sum_{\gamma \in C_{\Gamma}-\{1\}} \frac{\chi(\gamma) t_{\gamma}^{2 s} C(\gamma) \chi_{\sigma_{p}}\left(m_{\gamma}\right)}{\sqrt{\pi} \Gamma(s) j(\gamma)} \frac{K_{-s+\frac{1}{2}}\left(\alpha_{p} t_{\gamma}\right)}{\left(2 \alpha t_{\gamma}\right)^{s-1 / 2}}
\end{aligned}
$$

where $V_{\Gamma}=\chi(1) \operatorname{Vol}(\Gamma \backslash G) / 4 \pi$, and we have defined $\alpha_{p}^{2}:=b^{(p)}+\left(\rho_{0}-p\right)^{2}$,

$$
\xi_{n \ell} \stackrel{\text { def }}{=} \frac{(-1)^{\ell+1}\left(1-2^{-2 \ell-2 n-1}\right)}{n !(2 \ell+2 n+2)} B_{2 \ell+2 n+2} .
$$

Proof. For the identity component we get

$$
\zeta_{I}^{(N)}(s, p)=\frac{V_{\Gamma}}{\Gamma(s)} \int_{0}^{\infty} t^{s-1} d t \int_{\mathbb{R}} \mu_{\sigma_{p}} e^{-t\left(r^{2}+\alpha_{p}^{2}\right)} d r .
$$

For $\sigma_{p}$ the natural representation of $S O(2 k-1)$ on $\Lambda^{p} \mathbb{C}^{2 k-1}$, we have the corresponding HarishChandra-Plancherel density given - for a suitable normalization of the Haar measure $d x$ on $G-$ by

$$
\mu_{\sigma_{p}(r)}=\frac{\pi}{2^{4 k-4}[\Gamma(k)]^{2}}\left(\begin{array}{c}
2 k-1 \\
p
\end{array}\right) P_{\sigma_{p}}(r) r \tanh (\pi r),
$$

for $0 \leq p \leq k-1$, where

$$
P_{\sigma_{p}}(r)=\prod_{\ell=2}^{p+1}\left[r^{2}+\left(k-\ell+\frac{3}{2}\right)^{2}\right] \prod_{\ell=p+2}^{k}\left[r^{2}+\left(k-\ell+\frac{1}{2}\right)^{2}\right]
$$

is an even polynomial of degree $2 k-2$. We have that $P_{\sigma_{p}}(r)=P_{\sigma_{2 k-1-p}}(r)$ and $\mu_{\sigma_{p}}(r)=\mu_{\sigma_{2 k-1-p}}(r)$ for $k \leq p \leq 2 k-1$. Define the Miatello coefficients [13, 14] $a_{2 \ell}^{(p)}$ for $G=S O_{1}(2 k+1,1)$ by $P_{\sigma_{p}}(r)=$ $\sum_{\ell=0}^{k-1} a_{2 \ell}^{(p)} r^{2 \ell}, 0 \leq p \leq 2 k-1$. Replacing the Harish-Chandra-Plancherel measure, we obtain two representations for $\zeta_{I}^{(N)}(s, p)$, which holds for the cases of odd and even dimension. Using the identities $\tanh (\pi r)=1-2\left(1+e^{2 \pi r}\right)^{-1}$, and $\int_{0}^{\infty}\left(1+e^{2 \pi r}\right)^{-1} r^{2 \ell-1} d r=(-1)^{\ell-1}\left(1-2^{1-2 \ell}\right)(4 \ell)^{-1} B_{2 \ell}$, where $B_{\ell}$ is the $\ell$-th Bernoulli number, we get Eqs. (3.5) and (3.6). Finally using the following representation for the Bessel function [7] $K_{v}(z)=(1 / 2)(z / 2)^{v} \int_{0}^{\infty} e^{-t-\frac{z^{2}}{4 t}} t^{-v-1} d t(|\arg z|<\pi / 2$ and $\Re z^{2}>0$ ) we get formula (3.7). 


\section{References}

[1] M. J. Duff, B. E. Nilsson and C. N. Pope, Kaluza-Klein Supergravity, Phys. Rep. 130 (1986) 1. ${ }^{1}$

[2] A. A. Bytsenko, G. Cognola, L. Vanzo and S. Zerbini, Quantum Fields and Extended Objects in Space-Times with Constant Curvature Spatial Section, Phys. Rep. 266 (1996) 1 [hep-th/9505061].

[3] J. Maldacena, The Large N Limit of Superconformal Field Theories and Supergravity, Adv. Theor. Math. Phys. 2 (1998) 231 [hep-th/9711200].

[4] R. Aurich, S. Lustig, F. Steiner and H. Then, Hyperbolic Universes with a Horned Topology and the CMB Anisotropy, Class. Quant. Grav. 21 (2004) 4901 [astro-ph/0403597].

[5] N. Kaloper, J. March-Russell, G. D. Starkman and M. Trodden, Compact Hyperbolic Extra Dimensions: Branes, Kaluza-Klein Modes and Cosmology, Phys. Rev. Lett. 85 (2000) 928 [hep-ph/0002001].

[6] A. A. Bytsenko, G. Cognola and S. Zerbini, Finite Temperature Effects for Massive Fields in D-Dimensional Rindler-Like Spaces, Nucl. Phys. B458 (1996) 267 [hep-th/9508104].

[7] I. S. Gradshteyn and I. M. Ryzik, Table of Integrals, Series, and Products, Collected and Enlarged Edition Prepared by A. Jeffrey, Academic Press, New York, 1980.

[8] Yu. N. Obukhov, The Geometrical Approach to Antisymmetric Tensor Field Theory, Phys. Lett. B109 (1982) 195.

[9] D. Fried, Analytic Torsion and Closed Geodesics on Hyperbolic Manifolds, Invent. Math. 84 (1986) 523.

[10] E. Elizalde, S. D. Odintsov, A. Romeo, A. A. Bytsenko and S. Zerbini, Zeta Regularization Techniques with Applications, World Scientific, Singapore, 1994.

[11] F. L. Williams, Topological Casimir Energy for a General Class of Clifford-Klein Space-Times, J. Math. Phys. 38 (1997) 796.

[12] A. A. Bytsenko, G. Cognola, E. Elizalde, V. Moretti and S. Zerbini, Analytic Aspects of Quantum Fields, World Scientific, Singapore, 2003.

[13] R. Miatello, The Minakshisundaram-Pleijel Coefficients for the Vector-Valued Heat Kernel on Compact Locally Symmetric Spaces of Negative Curvature, Trans. Am. Math. Soc. 260 (1980) 1.

[14] A. A. Bytsenko, E. Elizalde and M. E. X. Guimarães, Operator Product on Locally Symmetric Spaces of Rank One and the Multiplicative Anomaly, Int. J. Mod. Phys. A18 (2003) 2179 [hep-th/0305031].

\footnotetext{
${ }^{1}$ see also the paper of A. A. Bytsenko, M. E. X. Guimrães and J. A. Helayël-Neto: Hyperbolic Space Forms and Orbifold Compactification in M-theory, which is published in this volume.
} 